\title{
RESEARCH
}

Open Access

\section{Regulatory coupling between long noncoding RNAs and senescence in irradiated microglia}

\author{
Anan $\mathrm{Xu}^{1 \dagger}$, Rong $\mathrm{Li}^{1+}$, Anbang Ren ${ }^{1 \dagger}$, Haifeng Jian${ }^{1}$, Zhong Huang ${ }^{1}$, Qingxing Zeng ${ }^{1}$, Baiyao Wang ${ }^{1}$, \\ Jieling Zheng ${ }^{1}$, Xiaoyu Chen ${ }^{1}$, Naiying Zheng ${ }^{1}$, Ronghui Zheng ${ }^{1}$, Yunhong Tian' ${ }^{1}$, Mengzhong Liu ${ }^{1,2}$, Zixu Mao $^{3}$, \\ Aimin $\mathrm{Ji}^{1 *}$ and Yawei Yuan ${ }^{1^{*}}$
}

\begin{abstract}
Background: Microglia have been implicated in the pathogenesis of radiation-induced brain injury (RIBI), which severely influences the quality of life during long-term survival. Recently, irradiated microglia were speculated to present an aging-like phenotype. Long noncoding RNAs (IncRNAs) have been recognized to regulate a wide spectrum of biological processes, including senescence; however, their potential role in irradiated microglia remains largely uncharacterized.

Methods: We used bioinformatics and experimental methods to identify and analyze the senescence phenotype of irradiated microglia. Western blotting, enzyme-linked immunosorbent assays, immunofluorescence, and quantitative real-time reverse transcription-polymerase chain reaction were performed to clarify the relationship between the radiation-induced differentially expressed IncRNAs (RILs) and the distinctive molecular features of senescence in irradiated microglia.

Results: We found that the senescence of microglia could be induced using ionizing radiation (IR). A mutual regulation mode existed between RILs and three main features of the senescence phenotype in irradiated microglia: inflammation, the DNA damage response (DDR), and metabolism. Specifically, for inflammation, the expression of two selected RILS (ENSMUST00000190863 and ENSMUST00000130679) was dependent on the major inflammatory signaling pathways of nuclear factor kappa B (NF-KB) and mitogen-activated protein kinase (MAPK). The two RILs modulated the activation of NF-KB/MAPK signaling and subsequent inflammatory cytokine secretion. For the DDR, differential severity of DNA damage altered the expression profiles of RILs. The selected RIL, ENSMUST00000130679, promoted the DDR. For metabolism, blockade of sterol regulatory element-binding proteinmediated lipogenesis attenuated the fold-change of several RILs induced by IR.

Conclusions: Our findings revealed that certain RILs interacted with senescence in irradiated microglia. RILs actively participated in the regulation of senescence features, suggesting that RILS could be promising intervention targets to treat RIBI.
\end{abstract}

Keywords: Microglia, Radiation, Senescence, LncRNA, Inflammation, DNA damage response, Metabolism

\footnotetext{
*Correspondence: jiaimin@gzhmu.edu.cn; yuanyawei@gzhmu.edu.cn

${ }^{\dagger}$ Anan Xu, Rong Li and Anbang Ren contributed equally to this work.

'Department of Radiation Oncology, Affiliated Cancer Hospital \& Institute of

Guangzhou Medical University, No 78, Hengzhigang Road, Yuexiu District,

Guangzhou 510095, Guangdong, People's Republic of China

Full list of author information is available at the end of the article
}

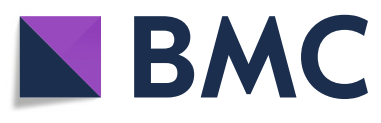

(c) The Author(s). 2020 Open Access This article is licensed under a Creative Commons Attribution 4.0 International License, which permits use, sharing, adaptation, distribution and reproduction in any medium or format, as long as you give appropriate credit to the original author(s) and the source, provide a link to the Creative Commons licence, and indicate if changes were made. The images or other third party material in this article are included in the article's Creative Commons licence, unless indicated otherwise in a credit line to the material. If material is not included in the article's Creative Commons licence and your intended use is not permitted by statutory regulation or exceeds the permitted use, you will need to obtain permission directly from the copyright holder. To view a copy of this licence, visit http://creativecommons.org/licenses/by/4.0/. The Creative Commons Public Domain Dedication waiver (http://creativecommons.org/publicdomain/zero/1.0/) applies to the data made available in this article, unless otherwise stated in a credit line to the data. 


\section{Background}

Ionizing radiation (IR), a fundamental treatment for intracranial and head-neck cancers, frequently causes irreversible and progressive radiation-induced brain injury (RIBI), especially cognitive deficit, which seriously influences a patient's quality of life (QoL) during long-term survival [1, 2]. Corticosteroids [3], donepezil [4], and memantine [5] are used to minimize cognitive deterioration; however, their effects are limited. Therefore, a thorough understanding of the pathogenesis of RIBI is urgently required to identify potential targets to prevent RIBI.

Microglia, the effector cells of innate immunity, are responsible for immune defense and brain homeostasis maintenance. In response to certain stimuli, microglia become active and provide beneficial functions that are essential for neuronal survival. However, once their activation passes the threshold of benefit and becomes deleterious, microglia switch to a neurotoxic phenotype and contribute to neuronal damage or death, which ultimately cause neurodegenerative diseases or RIBI [6, 7]. Interestingly, the functional changes of damageassociated molecular patterns (DAMPs)-stimulated microglia in the young adult brain are similar to the changes in normal aged microglia [8]. In addition, the neurotoxic phenotype of microglia exhibits various features of senescence $[9,10]$.

Cellular senescence, a process that imposes permanent proliferative arrest on cells in response to various stimuli, has emerged as a potentially crucial contributor to aging and age-related diseases. Senescent cells secrete numerous cytokines and matrix metalloproteases (MMPs) and sustain oxidative and genotoxic damage, which consequently contribute to tissue dysfunction and the aging process [11]. Following IR stimulation, the accumulation of DNA damage and the generation of oxidative stress are capable of triggering senescence in normal cells [12-15]. Murine neural stem cells could enter irreversible proliferative arrest, with features of senescence, after in vitro and in vivo IR treatment [12, 13]. In addition, astrocytes acquire senescence characteristics in primary culture conditions and in human brain tissue after exposure to IR [14]. Moreover, IR-induced senescence can be detected in brain microvascular endothelial cells [15]. Recently, it has been reported that the gene expression profile of irradiated primary microglia shares certain similarities with that in normal aging microglia [16]. Therefore, microglia might undergo senescence after IR stimulation; however, the mechanism of this transformation has not been characterized.

Several distinguishing molecular features have been emphasized in senescent cells, including senescenceassociated chronic inflammation, a prolonged DNA damage response (DDR), and altered metabolism [11]. Long noncoding RNAs (lncRNAs) comprise a large class of non-protein-coding transcripts with a length $>200$ bases [17]. Increasing evidence suggests their diverse roles in regulating the three characteristics of senescence. For example, in WI-38 fibroblasts, differentially expressed lncRNAs were compared between young and aged cells. Then, senescence-associated lncRNAs (SALRNAs) were identified, several of which are capable of regulating the onset of senescence [18]. In endothelial cells, lncRNA H19 ameliorates stress-induced senescence by counteracting inflammatory activation [19]. In THP1 macrophages, lncRNA THRIL promotes the transcription of the senescence-associated proinflammatory factor, transforming growth factor-alpha (TNF $\alpha)$ [20]. In HeLa cells, suppressor of Ty 6 homolog (SPT6) depletion induced lncRNAs that caused DNA damage, DNA replication stress, and eventually, senescence [21]. In human acute myeloid leukemia cells, lncRNA ANRIL inhibits cell senescence by repressing the expression of adiponectin receptor 1 (ADIPOR1), a key regulator of glucose metabolism [22]. Collectively, these data strongly support an essential role for lncRNAs in regulating senescence-associated inflammation, DDR, and metabolism. However, the involvement of lncRNAs in the IRinduced response of microglia remains uncharacterized.

In the present study, we confirmed that IR could induce the senescence of microglia and detected the three main features of irradiated microglia: inflammation, DDR, and metabolism. Altering the expression levels of radiation-induced differentially expressed lncRNAs (RILs) significantly modified the three distinguishing features of senescent microglia, and vice versa. The senescence-dependent regulation of RILs in irradiated microglia provides a method to avoid neuronal toxicity in response to IR.

\section{Methods \\ Cell culture}

The mouse microglial cell line BV2 and neuronal cell line HT22 were cultured in Dulbecco's modified Eagle's medium (DMEM) mixed with 10\% fetal bovine serum (Gibco, Grand Island, NY, USA) and incubated at $37^{\circ} \mathrm{C}$ in a $5 \% \mathrm{CO}_{2}$-humidified incubator.

Primary microglia were derived from mixed glial cultures using the "shaking off" method. Briefly, the brain stem, meninges, and cerebellum from neonatal (P0-1) $\mathrm{C} 57 \mathrm{Bl6} / \mathrm{J}$ mice were removed, and the remaining parts were chopped and digested using a mixture of papain (30 U/ml, Sigma-Aldrich, St. Louis, MO, USA) and Dnase I $(200 \mu \mathrm{g} / \mathrm{ml}$, Sigma-Aldrich $)$ at $37^{\circ} \mathrm{C}$ for $30 \mathrm{~min}$ in a $5 \% \mathrm{CO}_{2}$-humidified incubator. The cell suspension was collected and suspended in culture medium for glial cells (DMEM supplemented with $10 \%$ fetal bovine serum) and then cultured in a $75-\mathrm{cm}^{2}$ Falcon tissue culture flask coated with poly-D-lysine (PDL, $10 \mathrm{mg} / \mathrm{ml}$, 
Sigma-Aldrich) at $37^{\circ} \mathrm{C}$ in a $5 \% \mathrm{CO}_{2}$-humidified incubator. Half of the medium was changed after $8-12 \mathrm{~h}$ in culture and every 3 days thereafter, for a total culture time of 10-14 days. Microglia were shaken off from the primary glial cell culture $\left(220 \mathrm{rpm}, 37^{\circ} \mathrm{C} 2 \mathrm{~h}\right)$ with maximum yields between days 10 and 14. The floating cells were collected and seeded into a PDL-pretreated 24-well plate $\left(1 \times 10^{5}\right.$ cells per well in $\left.1 \mathrm{ml}\right)$ and incubated at $37^{\circ} \mathrm{C}$ in a $5 \% \mathrm{CO}_{2}$-humidified incubator.

\section{Isolation of adult primary microglia}

Adult male C57Bl6/J mice (6-8 weeks old) were used. The mice were sacrificed by anesthesia and perfused with ice-cold Dulbecco's phosphate-buffered saline (DPBS). The brain was placed in a culture dish with icecold D-PBS. The brain stem, meninges, and cerebellum were removed, and the remaining parts (maximum 500 $\mathrm{mg}$ ) were digested using $1980 \mu \mathrm{l}$ of enzyme mix (Miltenyi Biotec, Bergisch Gladbach, Germany). According to the manufacturer's protocol, the $1980 \mu$ l of enzyme mix contained $1950 \mu \mathrm{l}$ of enzyme mix 1 (enzyme P $50 \mu \mathrm{l}+$ buffer Z $1900 \mu \mathrm{l}$ ) and $30 \mu \mathrm{l}$ of enzyme mix 2 (enzyme A $10 \mu \mathrm{l}+$ buffer Y $20 \mu \mathrm{l})$. After removing the debris and red blood cells following the manufacturer's instructions, the cells were incubated with anti-CD11b (also known as integrin subunit alpha M (ITGAM)) microbeads (Miltenyi Biotec) at $4{ }^{\circ} \mathrm{C}$ for $15 \mathrm{~min}$. Magnetic separation was then used to separate the CD11b-positive population from the CD11b-negative population.

\section{lonizing radiation}

Adherent primary microglia or BV2 cells were irradiated at a distance of $100 \mathrm{~cm}$ from the source to the cells using a 6-MV linear accelerator (linac) (Clinac iX, Varian, Palo Alto, CA, USA) or a 225-KV X-ray irradiator (X-RAD 225, Precision X-Ray, Inc., North Branford, CT, USA), then returned to the $37^{\circ} \mathrm{C}, 5 \% \mathrm{CO}_{2}$ incubator. The dose-rate was $3 \mathrm{~Gy} / \mathrm{min}$ for the linac and $2 \mathrm{~Gy} / \mathrm{min}$ for the $\mathrm{X}$-ray irradiator.

Adult male C57Bl6/J mice (6-8 weeks old) were selected for irradiation. After anesthesia, the head of each mouse was placed in a $2 \times 2 \mathrm{~cm}^{2}$ treatment field, and a single dose of $10 / 20 \mathrm{~Gy}$ at a dose rate of $3 \mathrm{~Gy} / \mathrm{min}$ was delivered at a distance of $100 \mathrm{~cm}$ from the source to the skin using the Clinac iX. The mice were sacrificed at the indicated time after irradiation.

\section{Chemicals}

Oleic acid (OA) (Sigma-Aldrich) was mixed with $3 \mathrm{ml}$ of $0.1 \mathrm{mM} \mathrm{NaOH}$ (Shanghai Macklin Biochemical Co., Ltd, Shanghai, China) and then saponified at $75^{\circ} \mathrm{C}$ for 30 min. Next, the OA solution was mixed with $20 \%$ bovine serum albumin (BSA) (MRC, Shanghai, China), at $55^{\circ} \mathrm{C}$ for $30 \mathrm{~min}$. The stock solution of OA at $10 \mathrm{mM}$ in $10 \%$ BSA was then used to make the working solutions.

Lipopolysaccharide (LPS) was purchased from SigmaAldrich. Bay-11-7082, SP600125, U0126, and SB203580 were purchased from MedChemExpress LLC (Monmouth Junction, NJ, USA).

\section{Co-culture of BV2 and HT22 cells}

HT22 cells $\left(5.0 \times 10^{5}\right.$ cells per well $)$ were co-cultured with BV2 cells $\left(4 \times 10^{5}\right.$ cells per well) indirectly in 6well plate chambers (pore size $0.4 \mu \mathrm{m}$; Corning, NY, USA) to study the effect of IR-activated microglia on the survival of neuronal cells. In this co-culture system, irradiated BV2 communicated with HT22 through the semipermeable membrane, avoiding direct contact.

\section{Senescence assays}

A senescent cell staining kit (Solarbio, Beijing, China) was used to perform the senescence-associated- $\beta$-galactosidase (SA- $\beta$-gal) assay according to the manufacturer's protocol. The senescent BV2 cells $\left(5.0 \times 10^{5}\right.$ cells per well in 6-well plate) or primary microglia $\left(1 \times 10^{5}\right.$ cells per well in a 24-well plate) were identified as bluestaining cells under the microscope (Leica, Wetzlar, Germany). Total cells were counted in five random fields per culture dish to determine the percentage of SA- $\beta$ gal-positive cells.

The $\beta$-galactosidase activity of primary microglia was measured using a $\beta$-galactosidase activity assay kit (Solarbio) following the manufacturer's protocol. Onitrophenol was used as the standard solution. The standard, control, and sample were added to a 96-well plate and incubated at $37^{\circ} \mathrm{C}$ for $30 \mathrm{~min}$, followed by immunofluorescence. The absorbance of the solutions in the wells was measured at $400 \mathrm{~nm}$. The activity of $\beta$ galactosidase was calculated following the manufacturer's instructions.

\section{Immunofluorescence}

To label p16 ${ }^{\mathrm{INK} 4 \mathrm{~A}}$ (also known as cyclin-dependent kinase inhibitor 2A (CDNK2A)), non-irradiated/irradiated BV2 cells $\left(1 \times 10^{5}\right.$ cells per well in a 24 -well plate $)$ on glass coverslips were fixed using $4 \%$ paraformaldehyde (PFA) for $15 \mathrm{~min}$ and then permeabilized using $0.1 \%$ Triton X-100. Next, cells in the coverslips were washed with PBS and blocked in 3\% BSA for $30 \mathrm{~min}$. Rabbit anti-p16 ${ }^{\mathrm{INK} 4 \mathrm{~A}}$ primary antibody (Abcam, Cambridge, MA, USA) was used at 1:100 dilution, and goat antirabbit AlexaFluor-594 secondary antibodies (Abcam) were used at 1:200 dilution. 2-(4-amidinophenyl)-1H-indole-6-carboxamidine (DAPI); Sigma) staining was used before analysis under a fluorescence microscope (Leica).

To examine the co-distribution of $\mathrm{p} 16^{\mathrm{INK} 4 \mathrm{~A}}$ and Iba- 1 (ionized calcium-binding adapter molecule 1) in the 
same sample of primary microglia, a double immunofluorescence procedure was used. After fixation and permeabilization, the adherent non-irradiated/irradiated primary microglia $\left(1.0 \times 10^{5}\right.$ cells per well in a 24-well plate) were blocked in 3\% BSA for $30 \mathrm{~min}$ and then washed three times with PBS. The primary microglia were then incubated with goat anti-Iba-1 primary antibody (1:100, Novus biologicals, LLC, USA), and rabbit anti-p $16^{\mathrm{INK} 4 \mathrm{~A}}$ primary antibody $\left(1: 100\right.$, Abcam) at $4{ }^{\circ} \mathrm{C}$ overnight. The primary antibodies were detected by incubation of donkey anti-goat cy3-conjugation secondary antibody (1:200, Proteintech, Rosemont, IL, USA) and goat anti-rabbit AlexaFluor-594 secondary antibody (1: 200, Abcam) at room temperature for $1 \mathrm{~h}$. DAPI staining was used before analysis under a fluorescence microscope (Leica).

To examine the co-distribution of $\mathrm{p} 16^{\mathrm{INK} 4 \mathrm{~A}}$ and Iba-1 in the same mouse brain slice, a double immunofluorescence procedure was used. Before sacrifice, adult male C57Bl6/J mice were anesthetized and then perfused intracardially with $4 \%$ PFA. Brains were postfixed in $4 \%$ PFA at $4{ }^{\circ} \mathrm{C}$ overnight. Paraffin-embedded brain slides were then baked at $60{ }^{\circ} \mathrm{C}$ for $4 \mathrm{~h}$. The primary antibodies included in this study were Goat anti-Iba-1 antibodies (1:100, Novus biologicals) and rabbit anti-p16 ${ }^{\text {INK4A }}$ antibodies (1:100, Abcam). DAPI staining was used before analysis under a confocal laser-scanning microscope (Olympus, Tokyo, Japan).

To stain lipid droplets, the adherent non-irradiated/irradiated BV2 cells $\left(1.0 \times 10^{5}\right.$ cells per well in a 24-well plate) were washed twice with PBS and then lipiblue $(450-480 \mathrm{~nm})$ working solution (Dojindo Molecular Technologies Inc.) was added and incubated at $37^{\circ} \mathrm{C}$ for $30 \mathrm{~min}$ for immunofluorescence. Total cells were counted in five random fields per culture dish to determine the percentage of lipiblue-positive cells.

The fluorometric terminal deoxynucleotidyl transferase nick-end-labeling (TUNEL) assay was performed following the manufacturer's instructions (Promega, Madison, WI, USA). Cells were washed twice with PBS after fixing with $4 \%$ PFA for $30 \mathrm{~min}$ at $4{ }^{\circ} \mathrm{C}$ and then permeabilized in $0.2 \%$ Triton $\mathrm{X}-100$ for $5 \mathrm{~min}$, followed by rinsing with PBS. After the cells had equilibrated, the nucleotide mix and rTdT buffer were added incubated for $60 \mathrm{~min}$ at $37^{\circ} \mathrm{C}$ inside a humidified chamber avoiding direct light. Finally, after terminating the reaction using $2 \times$ SSC and PBS washing, DAPI staining was used before analysis under a fluorescence microscope (Leica).

\section{Flow cytometry}

The extent of apoptosis was determined by flow cytometry using an Annexin V-APC (Annexin VAllophycocyanin)/7AAD (7-Aminoactionomycin) assay (Multi sciences biotech, Co., Ltd, Hangzhou, China).
According to the manufacturer's instructions, after coculture with non-irradiated/irradiated BV2 cells, HT22 cells were washed twice with cold PBS, resuspended, and incubated with Annexin V-APC (dilution 1:40) and 7AAD (dilution 1:40) in the dark for $15 \mathrm{~min}$ at room temperature. For each experiment, $1 \times 10^{5}$ cells were analyzed.

\section{In situ hybridization}

Cells on glass coverslips $\left(1.0 \times 10^{5}\right.$ cells per well in a 24-well plate) were fixed using $4 \%$ PFA for $30 \mathrm{~min}$ and then permeabilized using $0.1 \%$ Triton X-100. The coverslips were then washed with PBS and soaked in a pre-hybridization buffer. The in situ hybridization (ISH) digoxigenin-labeled ENSMUST00000190863 or ENSMUST00000130679 probes (Boster, Pleasanton, CA, USA) were reconstituted in hybridization buffer $(50 \%$ formamide, $10 \%$ dextran sulfate, $2 \times$ TSSC, $0.01 \%$ sheared salmon sperm DNA, and $0.02 \%$ SDS), and incubated at $37{ }^{\circ} \mathrm{C}$ overnight. Cells were washed twice with $2 \times$ saline sodium citrate (SSC) for $5 \mathrm{~min}$ at $37^{\circ} \mathrm{C}$, twice with $0.5 \times \mathrm{SSC}$ for $15 \mathrm{~min}$ at $37^{\circ} \mathrm{C}$, and twice with $0.2 \times$ SSC for $5 \mathrm{~min}$ at $37^{\circ} \mathrm{C}$. After blocking with $5 \%$ normal goat serum for $1 \mathrm{~h}$ at $37^{\circ} \mathrm{C}$, the cells were incubated with antidigoxigenin alkaline phosphatase conjugate (Roche Applied Science, Basel, Switzerland) and stained with 5-bromo-4chloro-3-indolyl phosphate/nitro-blue tetrazolium chloride buffer for $4 \mathrm{~h}$. After DAPI staining, images were collected using a confocal microscope (Olympus).

\section{RNA-sequencing analysis}

Briefly, total RNA was extracted from non-irradiated/irradiated BV2 cells and purified using an RNeasy Mini Kit (Qiagen, Valencia, CA, USA). Next, the RNA samples were reverse transcribed into double-stranded DNA (cDNA) and labeled. RNA-seq analysis was performed by Sagene, Co. (Guangzhou, China) using the Illumina HiSeq $^{\text {Tm }} 2500$ platform. After analyzing the significance and false discovery rate (FDR), differentially expressed genes were chosen according to the $P$ value threshold and fold change. Functional profiling was performed using Gene Ontology (GO) and Kyoto Encyclopedia of Genes and Genomes (KEGG) databases.

\section{siRNA transfection}

Small interfering RNA (siRNA) targeting ENSMUST00000190863 or ENSMUST00000130679 and scrambled sequence (negative control, NC) were purchased from Generay (Shanghai, China). Cells were seeded on a 6-well plate and grown until they reached $50-60 \%$ confluence on the second day. Transfection was performed using Lipofectamine 3000 (Invitrogen Co., Carlsbad, California, US) according to the manufacture's instruction. The three target sequences for ENSMUST00000190863 were 5' -GAAGCACATATCCA CATTA-3', 5' -GCTAAGGACTAGGCCATAT-3' and 
5'-CCAGAGAACCAAAGAGAAA-3', respectively. The three target sequences for ENSMUST00000130679 were 5'-GCTTCACGCTTCACGCATA-3', 5' -CTTCCTTA GTCTACCATCA-3', and 5'-CAACTTCACGTTTCTC TAA- $3^{\prime}$, respectively.

\section{Construction of a recombinant virus}

H1-GFP-puro lentiviral vectors (synthesized by GenePharma) were used to knockdown the expression of ENSMUST00000190863 or ENSMUST00000130679 using short hairpin RNAs (shRNAs). A non-silencing shRNA cloned into the lentiviral vector was used as the negative control. The target sequences for ENSMUST00000190863 and ENSMUST00000130679

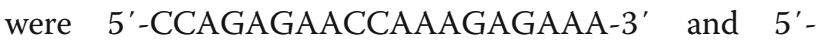
GCTTCACGCTTCACGCATA-3', respectively.

Quantitative real-time reverse transcription PCR (qRT-PCR) Total RNA was extracted using a Trizol reagent kit (Takara, Dalian, China), according to the manufacturer's instruction. After reversing transcription using a PrimeScript $^{\text {Ti }}$ RT reagent kit (Takara), quantitative real-time PCR (qPCR)was executed using a SYBR Premix Ex Taq ${ }^{\mathrm{mm}}$ kit (Takara) using gene-specific primers. The $2^{-\Delta \Delta C T}$ method was used to determine the fold changes. The sequences of the primers are shown in Supplementary Table 1.

\section{Cell fractionation}

Nuclear and cytoplasmic RNA from BV2 cells were fractionated using a purification kit (Norgenbiotek, ON, Canada). According to the manufacturer's protocol, after washing the cell monolayer with PBS, $200 \mu \mathrm{l}$ of ice-cold lysis buffer J was added to the 6-well culture plate for 5 $\mathrm{min}$, and then the lysate was transferred to an RNasefree microcentrifuge tube and centrifuged for $10 \mathrm{~min}$ at $15000 \mathrm{rpm}$. Next, $200 \mu \mathrm{l}$ of buffer SK was added to the supernatant (cytoplasmic RNA fraction) and $400 \mu \mathrm{l}$ of buffer SK was added to the pellet (nuclear RNA fraction). After vortexing, $200 \mu \mathrm{l}$ of $100 \%$ ethanol was added to each mixture. The mixtures were applied to a spin column, centrifuged for $1 \mathrm{~min}$ at $6000 \mathrm{rpm}$. The flowthrough was discarded, and $400 \mu \mathrm{l}$ of wash solution A was added to the column, which was centrifuged for 1 min three times. Next, $50 \mu$ l of elution buffer E was added to the column, which was centrifuged for $2 \mathrm{~min}$ at $2000 \mathrm{rpm}$ and $1 \mathrm{~min}$ at $14,000 \mathrm{rpm}$. The purified RNA sample was subjected to qRT-PCR analysis.

\section{Western blotting}

Cell lysates were harvested at the indicated times using radio-immunoprecipitation assay (RIPA) buffer (Cell signaling technology, CST, Danvers, MA, USA) containing phosphatase inhibitors and proteinase cocktails (Sigma-
Aldrich). Protein concentrations were measured using a bicinchoninic acid (BCA) protein assay kit (ComWin Biotech, Beijing, China). Equal amounts of proteins were separated using SDS-polyacrylamide gel electrophoresis, transferred to a polyvinylidene fluoride membrane, followed by reaction with primary antibodies. After reaction with labeled secondary antibodies, the target proteins were visualized using an ECL detection kit (Millipore, Billerica, MA, USA). The primary antibodies included in this study were rabbit anti-gamma $\mathrm{H} 2 \mathrm{~A}$ histone family member X ( $\gamma$ H2A.X) (1:2000, Abcam), rabbit anti-nuclear factor-kappa B p65 subunit (NFkB-P65 ${ }^{\mathrm{S} 536}$ ) (1:1000, SAB, College Park, MD, USA), rabbit antimitogen-activated protein kinase 14 (P38T-180 Y-182 MAPK) (1:1000, SAB), mouse anti-JUN $N$-terminal kinase (JNKT-183 Y-185) (1:1000, CST), rabbit anti-NFkBP65 (1:1000, CST), mouse anti-P38 MAPK (1:1000, Proteintech, Rosemont, IL, USA), mouse anti-JNK (1:1000, CST), rabbit anti-TP53 (1:1000, p53) (CST), rabbit antiglyceraldehyde-3-phosphate dehydrogenase (GAPDH) (1:1000, Proteintech), and rabbit anti- $\beta$-actin (1:1000, Proteintech).

\section{Enzyme-linked immunosorbent assay (ELISA)}

Cells were seeded into a 6-well plate. Culture supernatants were collected $24 \mathrm{~h}$ after IR exposure to detect the concentrations of TNF $\alpha$, IL-1 $\beta$, and IL-6. According to the manufacturer's instructions (Origene, Rockville, MD, USA), $100 \mu \mathrm{l}$ of standard, control, or samples were added to a 96 -well plate, and then $50 \mu$ l Biotin-labeled primary antibody was added to each well. After $2 \mathrm{~h}$ of incubation and four washes, $100 \mu \mathrm{l}$ of horseradish peroxidaseconjugated antibody was added to each well and incubated for $30 \mathrm{~min}$ in the dark. After washing four times, $100 \mu \mathrm{l}$ of $3,3^{\prime}, 5,5^{\prime}$-Tetramethylbenzidine (TMB) substrate was added to each well for $30 \mathrm{~min}$. Finally, $100 \mu \mathrm{l}$ of stop solution was added and the absorbance at 450 $\mathrm{nm}$ was determined using a microplate reader.

\section{Figure preparation and statistics}

Figures were prepared using GraphPad Prism 6.0 (GraphPad Software, Inc., La Jolla, CA, USA). Images were edited using Adobe Photoshop CS3 (Microsoft Corp., Redmond, WA, USA). The size of the sample for each sub-group was 5-8 (experiments in cell lines and primary cells were 5-8 wells per sub-group, in vivo experiments using mouse brains were 6 per sub-group), each experiment was repeated at least in triplicate, and the mean \pm standard deviation is presented. The Z-score was used for data normalization. Student's $t$ test and one-way analysis of variance (ANOVA) with Bonferroni's correction were used to compare the statistically significant differences between two groups and multiple 
groups, respectively. $P<0.05$ was considered statistically significant.

\section{Results \\ Irradiation induces microglial senescence in vitro}

It has been demonstrated that IR induces senescence in various cell types in the CNS [12-15]. In addition, certain similarities exist in the gene expression profiles of irradiated primary microglia and normal aging microglia [16]. To characterize cellular senescence in irradiated microglia, we examined the key senescent marker, senescence-associated- $\beta$-galactosidase (SA- $\beta$-Gal), in primary microglia and BV2 cells at $24 \mathrm{~h}$ after a single dose of $10 \mathrm{~Gy}$ delivered using a medical linac. The results showed much higher numbers of SA- $\beta$-Gal-stained cells after IR stimulation (Fig. 1a, b). Besides SA- $\beta$-Gal, the other key tool to identify cellular senescence is the expression of $\mathrm{p} 16^{\mathrm{INK} 4 \mathrm{~A}}$, which is a cyclin-dependent kinase inhibitor $(\mathrm{CDKi})$ that serves as a master regulator of cell cycle arrest [23]. Our study showed that the numbers of p16 ${ }^{\mathrm{INK} 4 \mathrm{~A}}$-positive primary microglia and BV2 cells increased dramatically at $24 \mathrm{~h}$ after $10 \mathrm{~Gy}$ delivered using the linac (Fig. 1c-f).

As presented in Fig. 1g, the level of p16 ${ }^{\mathrm{INK} 4 \mathrm{~A}}$ in BV2 cells began to increase at $24 \mathrm{~h}$ after exposure to $10 \mathrm{~Gy}$ and was constantly elevated at $48 \mathrm{~h}$. Two parallel yet interacting signaling pathways, p53-p21-retinoblastoma protein $(\mathrm{RB})$ and $\mathrm{p} 16^{\mathrm{INK} 4 \mathrm{~A}}-\mathrm{RB}$, participate in initiating and maintaining senescence [23]. Strikingly, the expression of p21 and p53 did not increase in the irradiated microglia (Fig. 1g-i). Therefore, we speculated that the p16 ${ }^{\text {INK4A }}-\mathrm{RB}$ pathway might be the main functional pathway that mediates senescence in irradiated microglia. In addition, the expression of matrix metalloproteinase 3 (MMP3), a classical marker of senescence [24], also increased dramatically at $24 \mathrm{~h}$ after IR (Fig. 1g). These results provided evidence that IR exposure induced microglial senescence in vitro.

\section{Irradiation induces microglial senescence in vivo}

To confirm the persistence of microglial senescence after irradiation in vivo, microglia from the cortex and hippocampus of irradiated adult male $\mathrm{C} 57 \mathrm{Bl6} / \mathrm{J}$ mice were isolated using a magnetic-activated cell sorting (MACS) technique (Fig. 2a). The sorting efficiency is presented in Fig. $2 \mathrm{~b}$. The result showed the continuous existence of activated $\beta$-galactosidase in isolated microglia at 14 and 30 days post-irradiation (Fig. 2c). The expression of the other key marker, p16 ${ }^{\mathrm{INK} 4 \mathrm{~A}}$, was also upregulated in microglia at 14 and 30 days post-irradiation in mouse brain slices (Fig. 2d, e). These results confirmed that IR induced persistence of senescence markers in microglia in vivo. Collectively, our results demonstrated that microglial senescence could be triggered by IR both in vitro and in vivo.

\section{The molecular features of senescence in irradiated microglia}

To identify the molecular features in irradiated microglia, we performed RNA-seq analysis based on nonirradiated/irradiated BV2 cells. Microglia are dysfunctionally activated by IR, which triggers inflammation and stress [25]. As expected, pathway analysis and functional annotation showed that seven out of the top 20 pathways were closely related to inflammation (Fig. 3a). Besides inflammation, biological network analysis showed that the gene set was also enriched for DDR and cellular metabolism (Fig. 3b), which are senescence features in neurodegenerative diseases [9]. Further evaluation of the transcriptome identified 316 significantly differentially expressed metabolism-related genes. Surprisingly, deeper exploration showed that a cluster of molecules consistent with those in neurodegeneration diseases was enriched (Fig. 3c, d), which added to the evidence that IR induces a senescence phenotype in microglia. Next, to verify the reliability of these three distinct features in irradiated microglia, upregulated genes in the transcriptome profile related to the NF-kB pathway (inflammation), apoptosis/cell cycle (DDR), and mitochondrial function (metabolism) were chosen for qRT-PCR confirmation. The result showed that the relative expression of these genes in BV2 cells was dramatically increased at $24 \mathrm{~h}$ after IR delivered using linac (Fig. 3e-g). Together, these results indicated that the three identified molecular features (inflammation, DDR, and metabolism) could represent the senescent phenotype of irradiated microglia.

\section{Differentially expressed LncRNAs in irradiated microglia}

A number of transcription factors and post-transcription factors have been implicated to drive senescence. As regulators of both transcriptional and post-transcriptional processes, lncRNAs have been demonstrated to have direct regulatory roles in senescence [18]; however, their roles in IR-induced senescence of microglia remain unexplored. Therefore, RNA-seq analysis of lncRNAs in non-irradiated/irradiated BV2 cells was carried out. We produced a volcano plot representing 10919 lncRNAs, of which 84 were upregulated (red plots) and 64 were downregulated (green plots) $(q<0.05)$ (Fig. 4a). Using more stringent criteria, the threshold for differential expression between the control and IR group was set as a $\log _{2}$ fold change $>2$ for lncRNAs. A total of 44 lncRNAs were identified, of which 26 were significantly upregulated and 18 were significantly downregulated. To validate the accuracy of RNA-seq data, qRT-PCR was used to test the expression of these RILs before and after IR. As shown in Fig. 4b, the changes in the expression levels of 


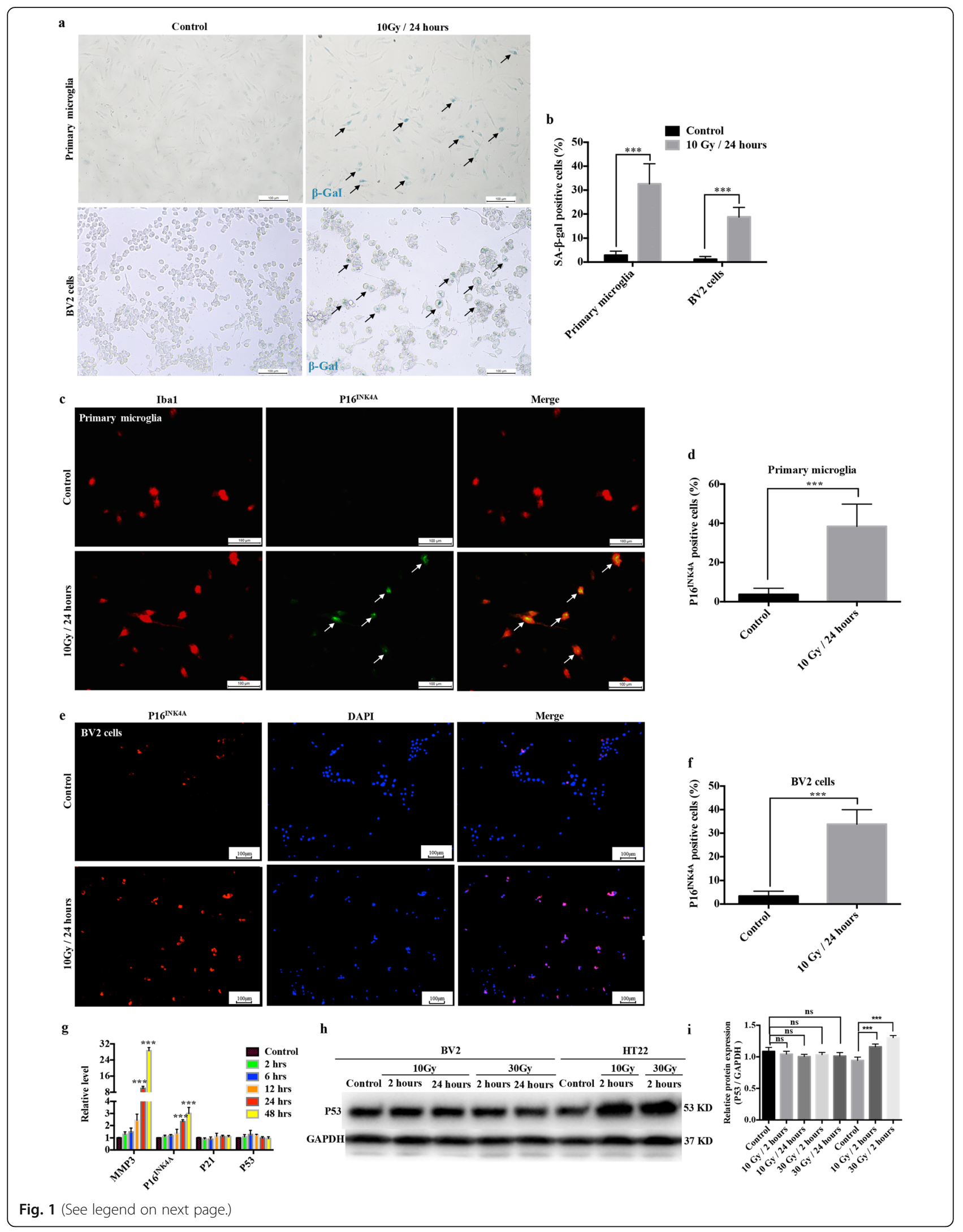


(See figure on previous page.)

Fig. 1 Irradiation induces microglial senescence in vitro. a Cytochemical staining for SA- $\beta$-gal in primary microglia and BV2 cells at $24 \mathrm{~h}$ after $10 \mathrm{~Gy}$ linac irradiation. Representative images are shown (scale bar, $100 \mu \mathrm{m}$ ). b The ratio of SA- $\beta$-gal positive cells is shown as the mean \pm SEM; $* * * P<0.001$, versus the control group. c Primary microglia were exposed to a single dose of IR (10 Gy) by linac. Cells were immunostained with anti-lba-1 (red) and anti-P16 ${ }^{\text {INK4A }}$ (green) antibodies $24 \mathrm{~h}$ after IR. Representative images are shown (scale bar, $100 \mu \mathrm{m}$ ). $\mathbf{d}$ The ratio of P16 $6^{\text {INK } 4 \mathrm{~A}}$ positive primary microglia is shown as the mean \pm SEM; ${ }^{* *} P<0.001$, versus the control group. e BV2 cells were exposed to a single dose of IR (10 Gy) by linac. Cells were immunostained with anti-P16 $6^{\mathrm{INK} 4 \mathrm{~A}}$ antibody (red) $24 \mathrm{~h}$ after IR. Representative images are shown (scale bar, $100 \mu \mathrm{m}$ ). $\mathbf{f}$ The ratio of P16 $6^{\mathrm{INK} 4 \mathrm{~A}}$ positive BV2 cells is shown as the mean $\pm \mathrm{SEM}$; ${ }^{* * *} P<0.001$, versus the control group. $\mathbf{g}$ Expression levels of Mmp3, Cdkn2a (P16 $\left.{ }^{\mathrm{INK} 4 \mathrm{~A}}\right), \mathrm{Cdkn1a}(\mathrm{P} 21)$, and p53 in BV2 cells at different time points after 10 Gy IR by linac were examined using qRT-PCR (mean \pm SEM, ${ }^{* * *} P<0.001$ ). $\mathbf{h}$ The protein levels of p53 from BV2 cells and HT22 cells after exposure to different doses at different time points, as examined using western blotting. $\mathbf{i}$ Histogram of the quantification of the immunoreactive protein bands on the western blots (mean \pm SEM, ns, not significant, ${ }^{* * *} P<0.001$ )

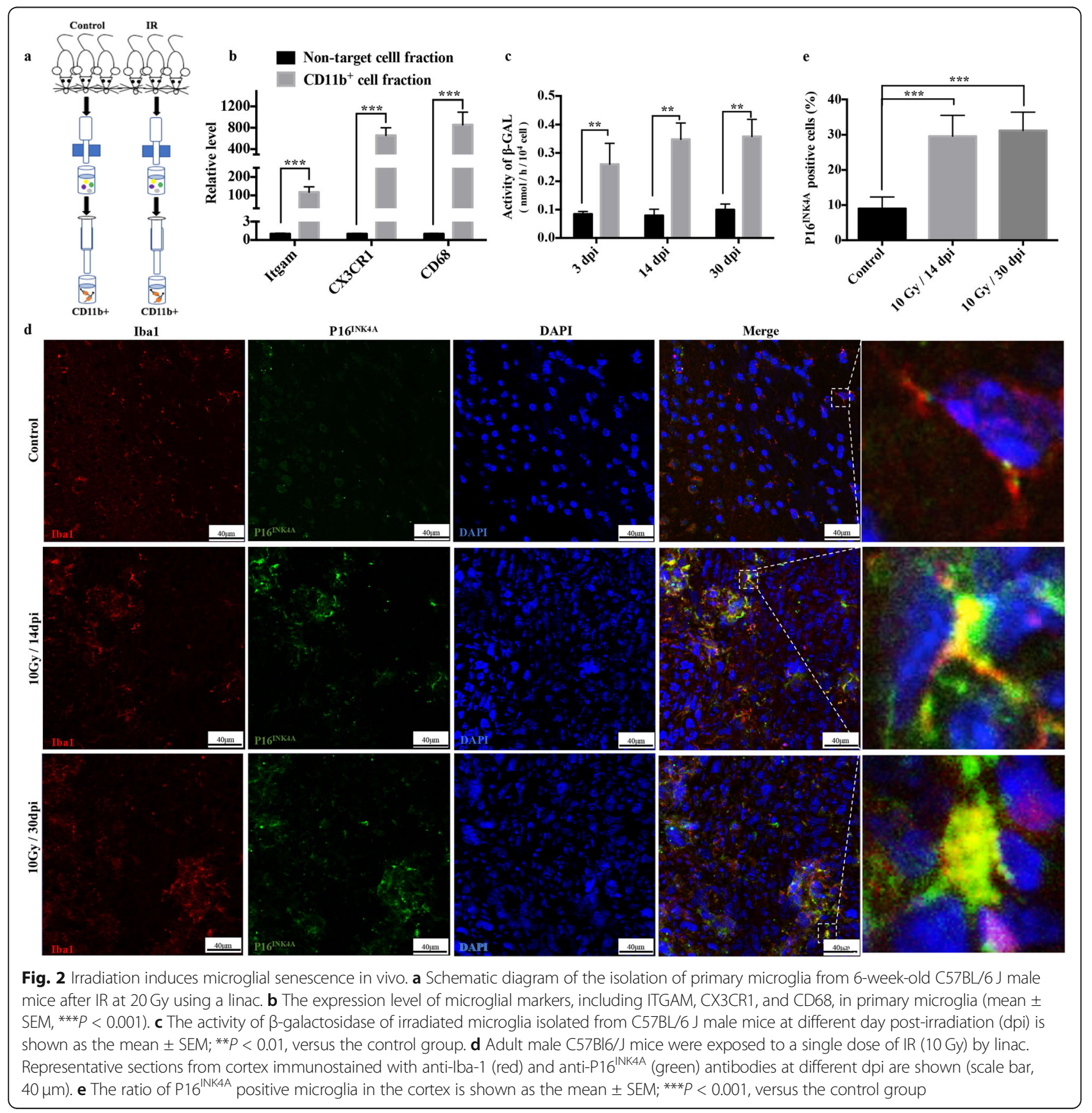




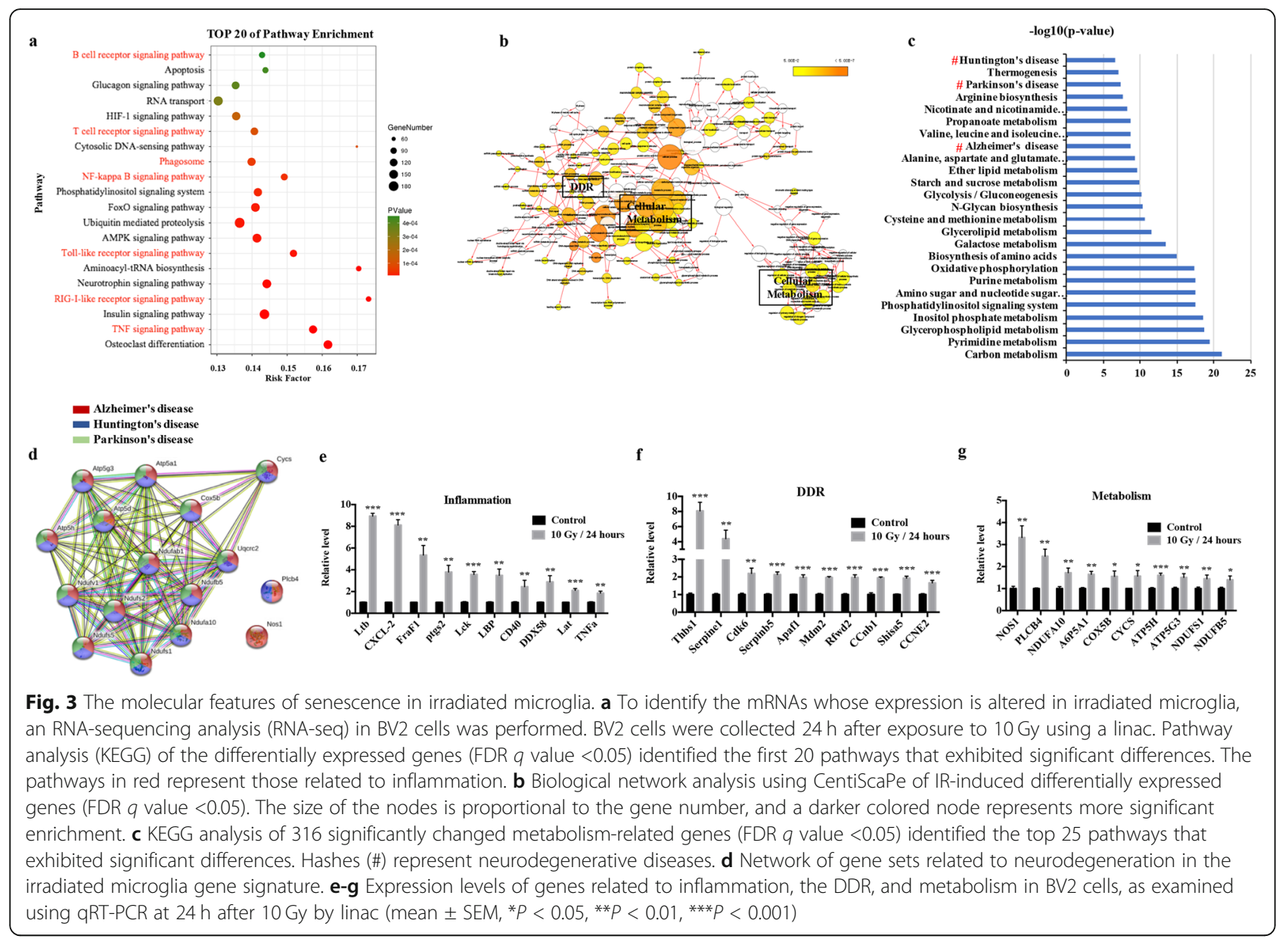

these IncRNAs were consistent with the RNA-seq profile. Next, two upregulated RILs, ENSMUST00000190863 and ENSMUST00000130679, were selected, both of which were located in the nucleus and cytoplasm of microglia (Fig. 4c, d). In addition, to characterize whether the selected RILs were functional in vivo, primary microglia were isolated from adult mice at $24 \mathrm{~h}$ after $20 \mathrm{~Gy}$ IR. Both RILs showed significantly increased expression in irradiated microglia (Fig. 4e), suggesting that these two RILs contributed to senescence in vivo. These data indicated that the selected RILs might be functional in the senescent process of irradiated microglia.

\section{The reciprocal relationship between inflammation and the RILs}

We next investigated whether the RILs can interplay with inflammation, the first identified feature of senescence in irradiated microglia. First, we measured the expression levels of TNF $\alpha$, interleukin (IL)-1 $\beta$, and IL-6 after treatment with different doses of IR delivered using linac at different time points. IR remarkably enhanced the mRNA expression levels of Tnfa, Il1b, and Il6 in a dose- and time-dependent manner (Fig. 5a). In addition, previous studies proved that the inflammatory state of microglia after IR activation was related to distinct signaling pathways, such as the NF- $\mathrm{kB}$ and MAPK pathways [26, 27]. Therefore, selective pharmacological inhibitors of the NF- $\mathrm{KB}$ pathway (Bay-11-7082) and the three main branches of MAPK pathway, including SP600125 (c-Jun N-terminal kinase, JNK), U0126 (extracellular signal-regulated kinase, ERK), and SB203580 (p38) were used to detect the possible pathways that act on the expression of the two selected RILs. The result showed that pretreatment with Bay-11-7082 (NF-kB), SP600125 (JNK), and SB203580 (p38) exerted partial inhibitory effects on IR-induced RIL expression (Fig. 5b). These findings suggested that the NF- $\mathrm{kB}$, JNK, and p38 pathways are responsible for the upregulation of the selected RILs in irradiated microglia.

Next, we investigated whether the selected RILs have a bearing on the activation of the NF-KB, JNK, or p38 pathways and further influence the release of proinflammatory cytokines. As presented in Fig. 5e, f, the phosphorylation levels of critical signaling proteins $\mathrm{P} 65$ (P-P65), JNK (P-JNK), and p38 (P-P38) were significantly upregulated after $10 \mathrm{~Gy}$ delivered using a linac in the 


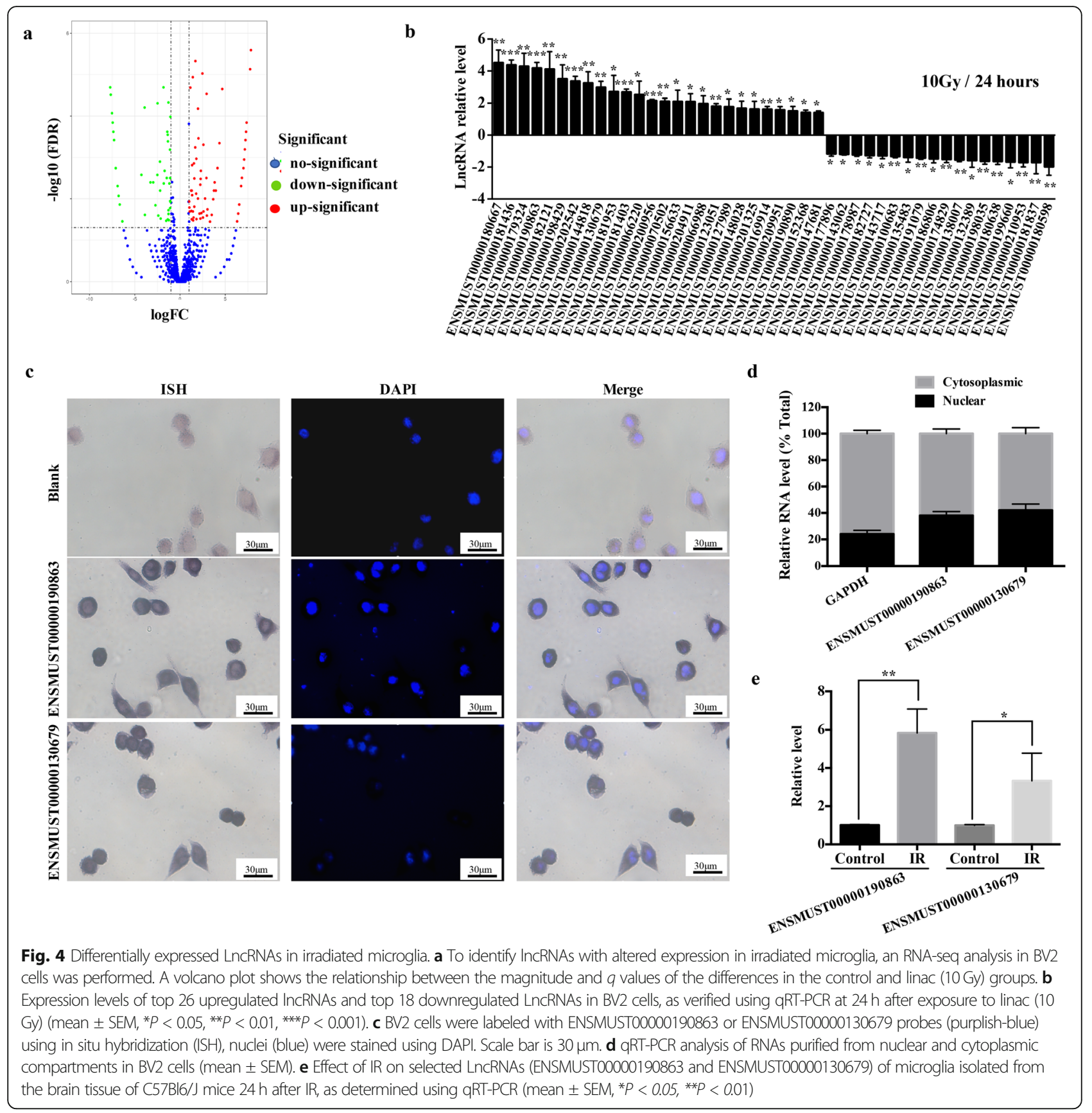

treatment of BV2 cells. Importantly, silencing the expression of ENSMUST00000190863 attenuated the phosphorylation level of P65, while silencing the expression of ENSMUST00000130679 decreased the phosphorylation level of P65, JNK, and P38 induced by IR (Fig. 5e, f). Furthermore, silencing of the two selected RILs did not change the basal levels of inflammatory cytokines (Fig. 5g); however, knockdown of ENSMUST00000190863 significantly decreased the level of TNF $\alpha$ triggered by IR (Fig. $5 \mathrm{~g}$ left), while the levels of IL-1 $\beta$ and IL- 6 decreased significantly after
ENSMUST00000130679 was knocked down in the IR group (Fig. $5 \mathrm{~g}$ middle and right). These data indicated that the inflammatory state affected the expressions of RILs, while conversely, the RILs could regulate the phosphorylation level of critical signaling proteins in the IR-induced inflammatory process and the downstream release of pro-inflammatory cytokines.

The reciprocal relationship between the DDR and RILs Different types of radiation with different energy levels vary in their linear energy transfer (LET) properties. 


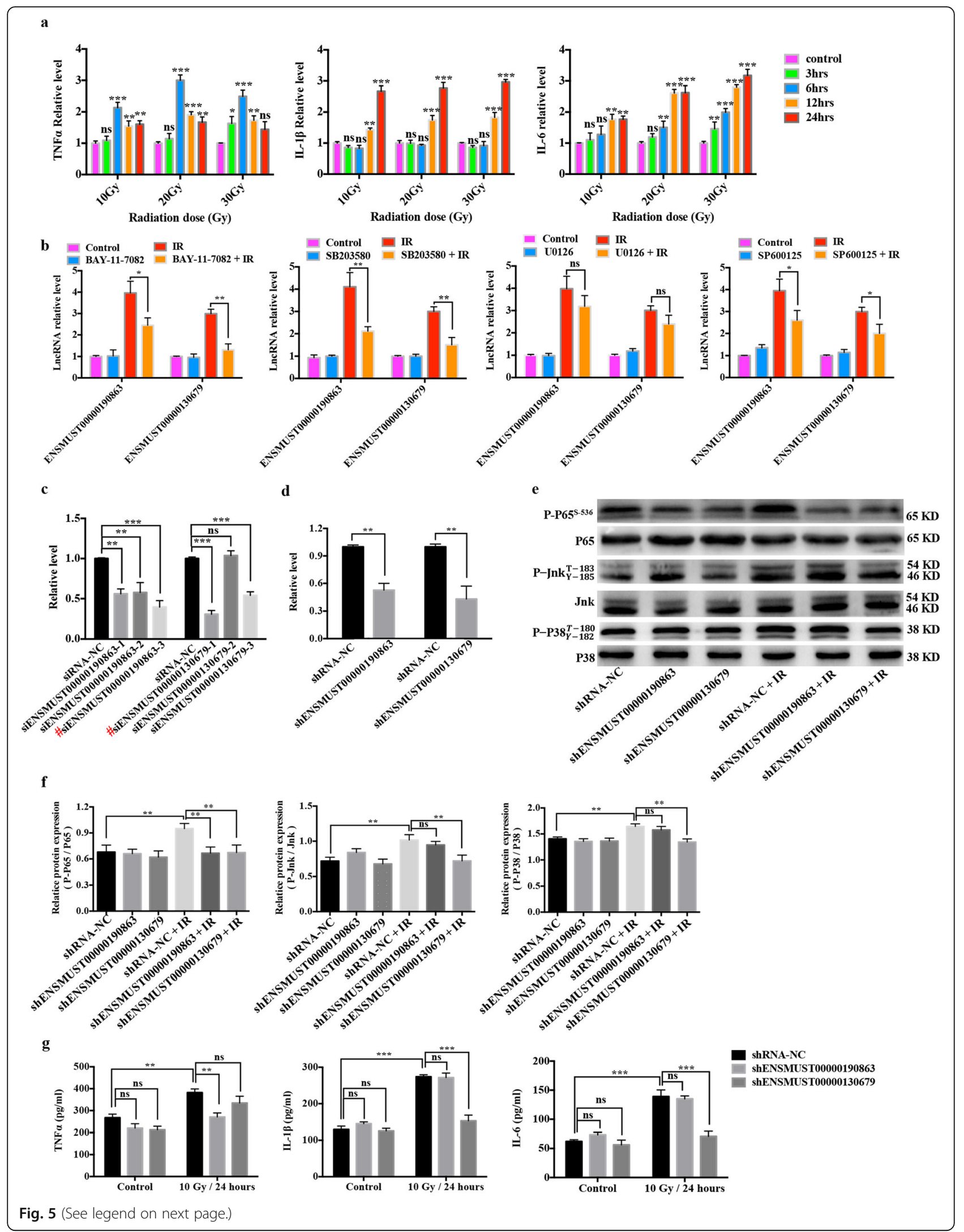


(See figure on previous page.)

Fig. 5 The reciprocal relationship between inflammation and the RILs. a Inflammatory cytokine expression in BV2 cells treated with 10 Gy, 20 Gy, and $30 \mathrm{~Gy}$ IR using a linac at different time points (mean \pm SEM, ns, not significant, ${ }^{*} P<0.05$, ${ }^{*} P<0.01,{ }^{* *} P<0.001$ ). b BV2 cells were

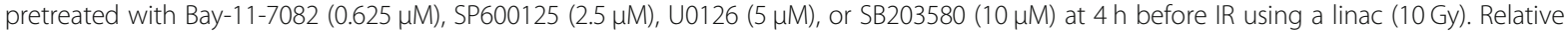
expression levels of IncRNAs ENSMUST00000190863 and ENSMUST00000130679 were evaluated at $24 \mathrm{~h}$ after IR using qRT-PCR (mean \pm SEM, ns, not significant, ${ }^{*} P<0.05,{ }^{*} P<0.01$ ). c qRT-PCR assay of the efficacy of expressing siRNA targeting RILs or NC in BV2 cells (mean \pm SEM, ns, not significant, ${ }^{* *} P<0.01,{ }^{* *} P<0.001$ ). Hash (\#) represents the most effective sequences (ENSMUST00000190863-3 and ENSMUST00000130679-1) which were chosen for further study. $\mathbf{d}$ The chosen sequences were used to construct of recombinant virus. qRT-PCR assay of the efficacy of expressing shRNA targeting RILs or NC in BV2 cells (mean \pm SEM, ${ }^{* *} P<0.01$ ). e The phosphorylation levels of P65 (P-P65), Jnk (P-Jnk), and p38 (PP38) after treatment with shRNAs targeting RILs or NC at $4 \mathrm{~h}$ after exposure to $10 \mathrm{~Gy}$ using a linac in BV2 cells, as revealed using western blotting. $\mathbf{f}$ Histogram of the quantification of the immunoreactive protein bands on the western blots (mean $\pm \mathrm{SEM}$, ns, not significant, ${ }^{* *} P<0.01$ ). $\mathbf{g}$ The effects of ENSMUST00000190863 or ENSMUST00000130679 knockdown on the expression levels of inflammatory cytokines (TNFa, IL-1 3 , and IL-6) secreted by BV2 cells at $24 \mathrm{~h}$ after $10 \mathrm{~Gy}$ IR using a linac, as measured using ELISA (mean \pm SEM, ns, not significant, ${ }^{* * P} P<0.01,{ }^{* * *} P<0.001$ )

With increasing LET, the severity of the DDR increases [28]. To investigate whether RILs can interplay with the DDR, the second identified feature of senescence in radiated microglia, we used different radiation energies at the same dose to trigger different DDRs. As expected, compared with the $225 \mathrm{KV}$ X-ray irradiator, BV2 cells accumulated higher levels of the DDR marker protein $\gamma \mathrm{H} 2 \mathrm{~A}$.X when using a $6 \mathrm{MV}$ linac (Fig. 6a, b), indicating that a more severe DDR is induced at higher radiation energy. To investigate the impact of different DDR levels on the expression of RILs, seven upregulated and three downregulated RILs, including the selected

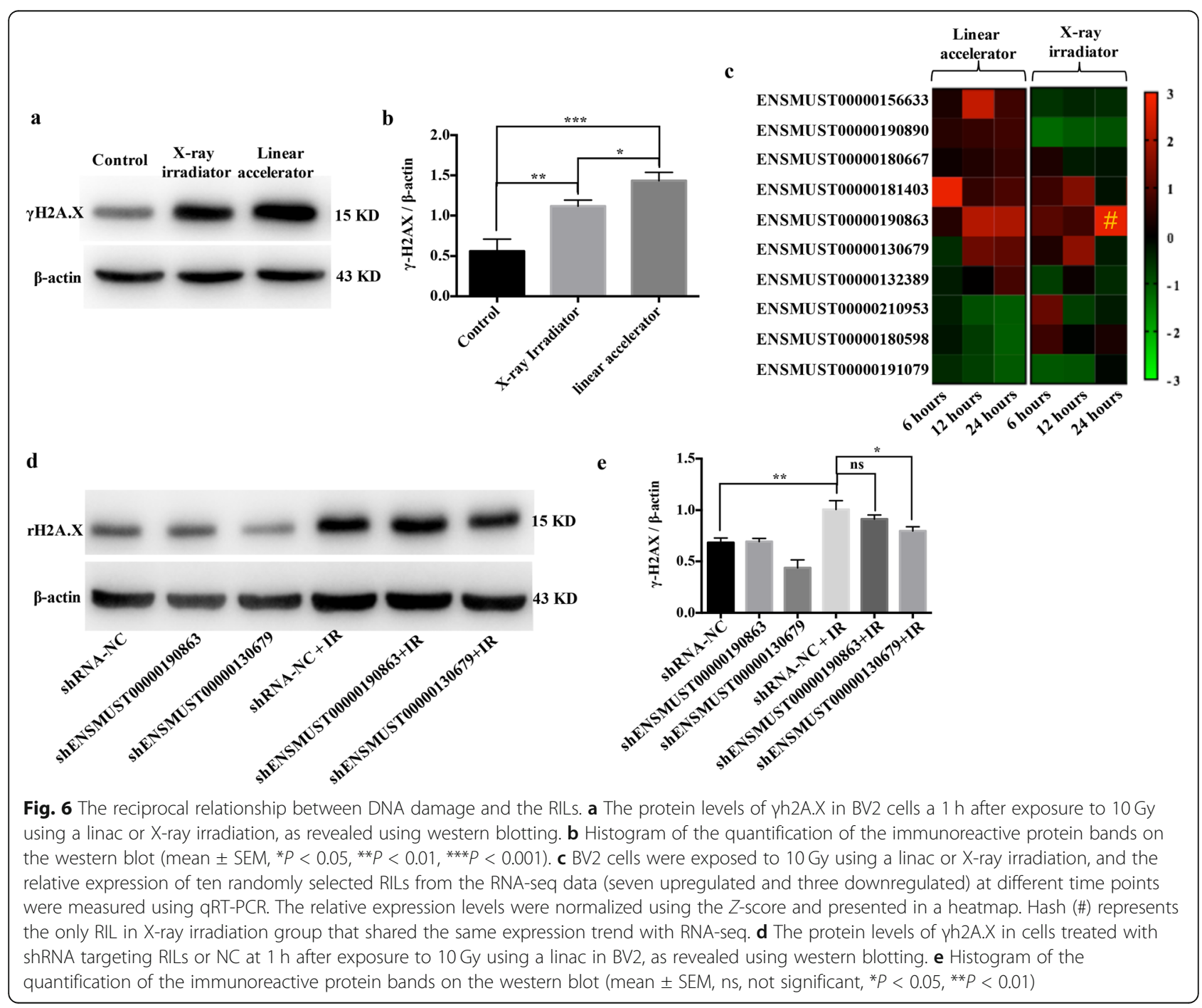


ENSMUST00000190863 and ENSMUST00000130679, were chosen and validated using qRT-PCR. As shown in the heatmap (Fig. 6c), all these candidates showed the same expression profile after higher radiation energy exposure using linac at $24 \mathrm{~h}$ between the RNA-seq and qRT-PCR data (Fig. 6c left); however, only ENSMUST00000190863 had the same trend after lower radiation energy exposure using the X-ray irradiator (Fig. 6c right). One possible explanation is that different severities of DDR would result in variations in the expression profiles of the RILs.

To identify the possible regulatory roles of the two selected RILs in the severity of DDR, western blotting was performed. The upregulation of $\gamma \mathrm{H} 2 \mathrm{~A}$.X triggered by $10 \mathrm{~Gy}$ using linac was attenuated when ENSMUST00000130679 was silenced, but not when ENSMUST00000190863 was silenced (Fig. 6d, e). These results demonstrated that the severity of DDR affected the expression of RILs. In turn, certain RILs have the ability to influence the severity of DDR.

\section{The reciprocal relationship between lipid metabolism and the RILs}

The third identified molecular feature of senescence in irradiated microglia was cellular metabolism. Recent studies showed that lipid droplets (LDs) accumulate in microglia during the aging process $[29,30]$. To detect whether LD accumulation also occurs in IR-induced senescent microglia, we exposed BV2 cells to a 10 Gy single-dose delivered using linac and examined LDs using lipiblue staining. The result showed a significantly increased number of LDs in irradiated BV2 cells compared with that in the control cells, similar to those stimulated by OA as a positive control. Surprisingly, after pretreatment with betulin, a sterol regulatory elementbinding protein (SREBP) inhibitor, the number of LDs in irradiated BV2 cells decreased significantly (Fig. 7a, b). This suggested that the accumulation of LDs in microglia is caused by the activation of lipogenesis after IR. Next, qRTPCR was used to test whether lipogenesis affected the expression of RILs. As shown in Fig. 7c, the lipogenesis inhibitor betulin significantly attenuated the expression changes of RILs triggered by IR (including ENSMUST00000190863 and ENSMUST00000130679).

LncRNAs frequently exert their biological functions by regulating the expression of nearby genes [31]. Therefore, we examined 3589 neighboring genes using KEGG analysis and identified 226 genes related to cellular metabolism (Fig. 7d). Among them, 79 were lipid metabolismrelated, and more than half of these (45/79) were lipogenesis- related (Fig. 7e). Collectively, our data suggested that lipogenesis is activated by IR, which triggers changes in the expression levels of RILs. In turn, RILs might function in lipogenesis via the effects of their neighboring genes.

\section{Blockade of the RILs alleviated neuronal damage after radiation}

To determine whether inhibiting the expression of the two selected RILs associated with senescence in IR-

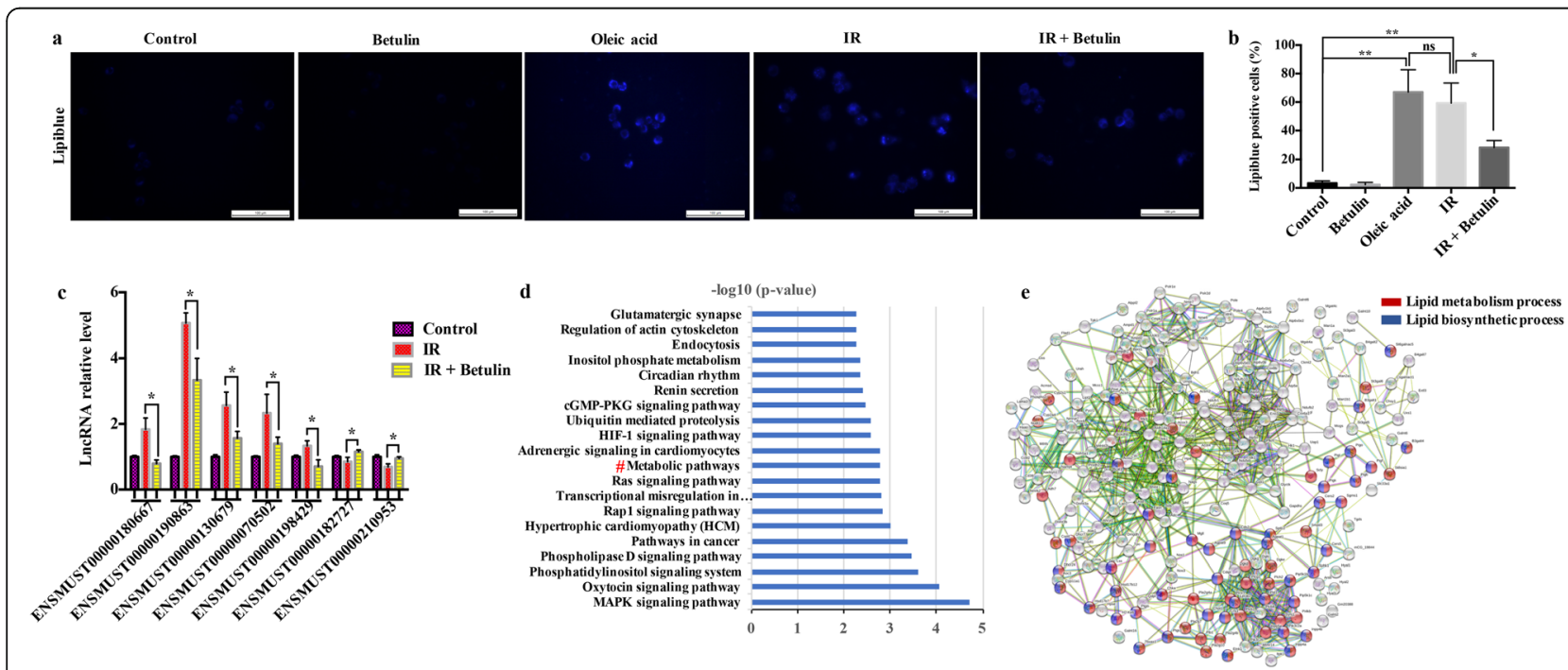

Fig. 7 The reciprocal relationship between lipid metabolism and the RILs. a BV2 cells were exposed to $10 \mathrm{~Gy}$ using a linac at $5 \mathrm{~h}$ after treatment with/without $2 \mu \mathrm{M}$ betulin. Representative micrographs of lipiblue staining in BV2 cells at $24 \mathrm{~h}$ after IR exposure. $100 \mu \mathrm{M}$ Oleic acid (OA) treatment was used as the positive control. Scale bar $=100 \mu \mathrm{m}$. b Quantification of lipiblue positive cells (mean \pm SEM, ns, not significant, ${ }^{*} P<0.05,{ }^{*} P<$ 0.01). c The relative expression levels of randomly selected RILs in BV2 cells at $24 \mathrm{~h}$ after exposure to $10 \mathrm{~Gy}$ using a linac with/without betulin $(2 \mu \mathrm{M})$ (mean $\left.\pm \mathrm{SEM},{ }^{*} P<0.05\right)$. d KEGG was used to cluster 3589 neighboring protein genes of RILs (neighboring genes within 20 kilobase of the 84 upregulated and 64 downregulated RILs (FDR q value <0.05) in RNA-seq data). Hashes (\#) represent 226 metabolism-related genes. e GO analysis identified 79 lipid metabolism-related genes (red), and 45 lipid biosynthetic metabolism-related genes (blue) from the RILs' neighboring protein genes 
induced microglia could affect neuronal damage, irradiated BV2 cells were co-cultured with the mouse neuron cell line HT22 in a Transwell system. Apoptotic neuronal cells were detected using TUNEL staining and quantified using flow cytometry. The apoptosis ratio of HT22 cells increased remarkably after co-culture with irradiated BV2 cells (Fig. 8a-c). Surprisingly, this ratio decreased dramatically when ENSMUST00000190863 or ENSMUST00000130679 were inhibited (Fig. 8b, c). In summary, these findings indicated that neuronal damage caused by irradiated microglia could be alleviated by downregulating the expression of certain RILs.

\section{Discussion}

In this study, we showed that microglia acquired a senescence phenotype after IR. Analysis of the transcriptome of irradiated microglia identified three main molecular features of senescence, including inflammation, the DDR, and metabolism. Our findings also showed that RILs are closely related to the molecular features of the senescence phenotype in irradiated microglia (Fig. 8d).

The molecular characteristics of primary microglia at $24 \mathrm{~h}$ and 1-month post-irradiation in adult mice share similarities with primary microglia in aging mice [16]. In this study, we found that the two key markers of senescence, SA- $\beta-$ Gal and $\mathrm{p} 16^{\mathrm{INK} 4 \mathrm{~A}}$, were both elevated dramatically in irradiated microglia. We also observed the persistence of $\beta$-galactosidase activity and the p16 ${ }^{\text {INK4A }}$ signal in microglia at 1 -month post-irradiation in vivo. Chronic senescence is believed to be a driver of age-related tissue dysfunction. The gradual accumulation of senescent cells alters the physiological function of tissues and organs, ultimately leading to age-related diseases [23, 32]. Microglial senescence has profound consequences for neuronal activity and cognitive function in the normal aging brain [10], and irradiated microglia play a pivotal role in the underlying pathology of RIBI [7]; therefore, we speculated that the senescence induced in irradiated microglia might also explain the observed neurotoxic effects. In addition, the two main signaling pathways, $\mathrm{p} 53-\mathrm{p} 21-\mathrm{RB}$ and $\mathrm{p} 16^{\mathrm{INK} 4 \mathrm{~A}}-\mathrm{RB}$, are coordinated to initiate and maintain senescence [23]. Interestingly, we detected a significant increase in $\mathrm{p} 16^{\mathrm{INK} 4 \mathrm{~A}}$ in irradiated microglia, whereas the expression levels of p53 and p21 remained unchanged. In addition, the p53 signal was not detected after DNA damage in irradiated astrocytes [33]. Furthermore, a significant role of $\mathrm{p} 16^{\mathrm{INK} 4 \mathrm{~A}}$ was demonstrated in $\mathrm{p} 53$ independent senescence of primary human fibroblasts [34]. Accordingly, we deduced that cellular senescence in irradiated microglia occurs in a p53-independent manner and that $\mathrm{p} 16^{\mathrm{INK} 4 \mathrm{~A}}-\mathrm{RB}$ signaling might be the major functional pathway.

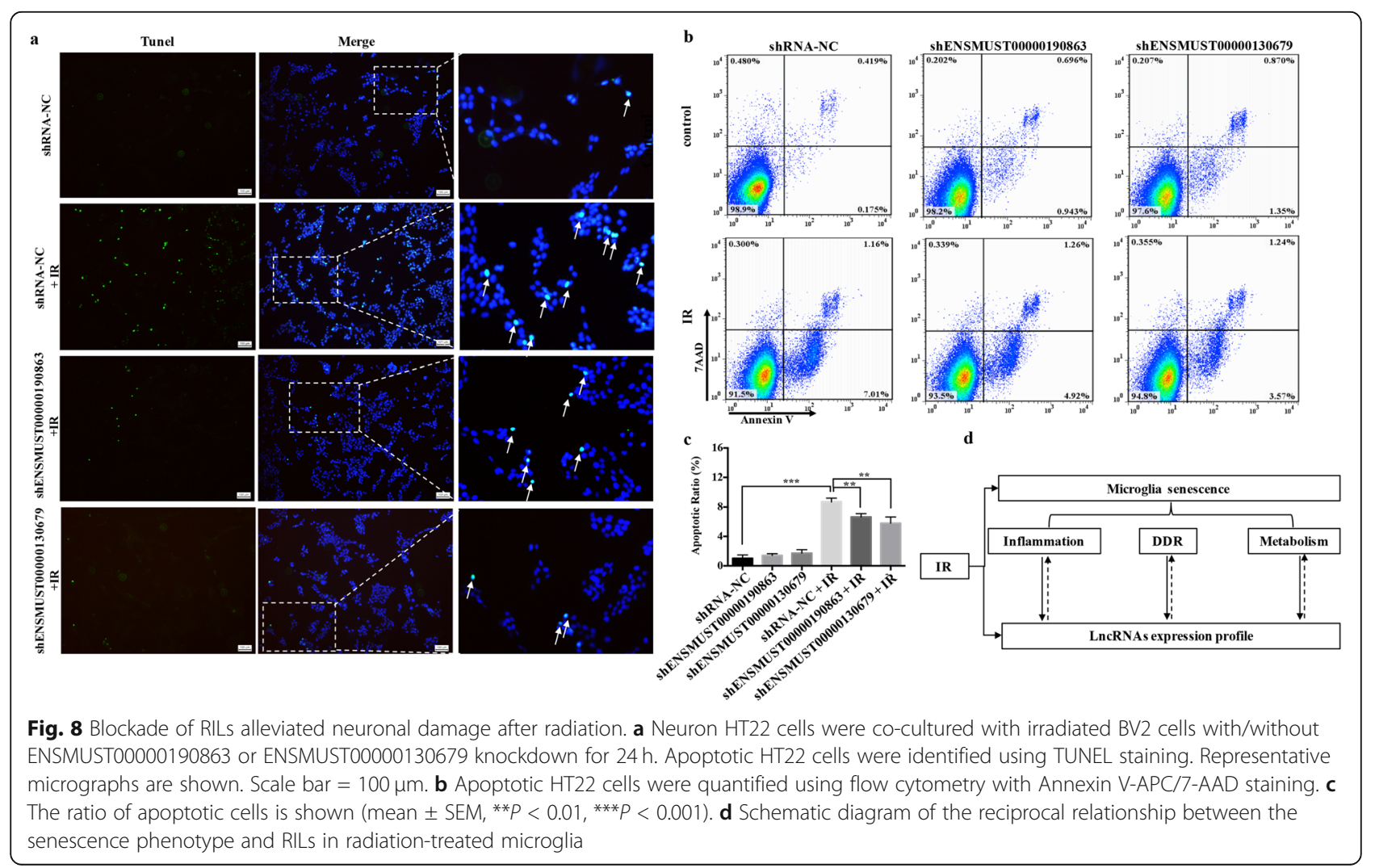


Increasing evidence has demonstrated sex-specific differences in normal aging microglia [35]. Consistently, sex-specific differences also exist in irradiated microglia, including microglia-mediated-inflammation, synaptic modification, and cognitive deficit [36, 37]. For example, the sex-specific difference in irradiated microgliamediated dendritic spine pruning was derived from a sex-specific difference in the expression of complement receptor 3 (CR3) [37]. We speculated that the phenotype of irradiation-induced microglial senescence found in the current study might also vary with sex, which is worthy of further exploration.

In senescent cells, characteristic molecular features have been observed [9]. In the present study, we confirmed the three main molecular features of senescence in the transcriptome of irradiated microglia as inflammation, the DDR, and metabolism. Sustained abnormal activation of microglia is thought to contribute to a chronic inflammatory state in the brain [38], which shares similarity with the role of microglia in neurodegenerative diseases [6]. In the present study, we demonstrated that the expression of genes related to NF- $\mathrm{kB}$, the central regulator of inflammation, were upregulated significantly after IR treatment. Secondly, a sustained DDR could be detected 1 month after IR in primary microglia [16]. Typically, DNA damage is resolved within hours; however, severe or irreparable DNA damage causing persistent DDR signaling is essential to establish and maintain a senescent phenotype [39]. Therefore, we measured the expression level of target genes of apoptosis and the cell cycle, the downstream mediators of DDR signaling, and demonstrated that the expression levels of these genes remained upregulated in microglia at $24 \mathrm{~h}$ after IR exposure. Thirdly, aged brains suffer from metabolic reprogramming [40]. Given the critical role of mitochondria in regulating metabolism, we examined the expression of genes related to mitochondrial function in the microglia and observed their increased expression after IR. Taken together, these findings provide evidence that IR induces changes to inflammation, the DDR, and metabolism that are senescence-specific.

LncRNAs have emerged as major regulators of genomic functions during aging [41]. In addition, senescenceassociated lncRNAs (SAL-RNAs) play direct regulatory roles in senescence [18]. Moreover, lncRNAs are abundantly expressed in cells of the CNS [42]. However, the function of RILs in senescent microglia remains unexplored. Our result revealed that the expression levels of the two selected RILs were increased significantly in irradiated microglia, suggesting their potential roles in regulating gene expression during senescence in irradiated microglia.

As an important feature of microglia senescence, inflammation is crucial in IR-induced RIBI [38] and agingrelated diseases [6]. Abnormally activated microglia constantly produce neurotoxic cytokines, such as IL-1 $\beta$,
TNF $\alpha$, and IL-6, and increase the level of reactive oxygen species (ROS), which consequently induce oxidative stress, ultimately leading to DNA damage [25]. In the present study, inflammatory cytokines were released persistently by microglia after IR and increased in an IR dose-dependent manner. In addition, in peripheral macrophages, lncRNAs are induced or suppressed after the activation of intracellular signaling pathways, and these alterations to IncRNA levels promote, restrain, or suppress the response of immunity-related genes [43-45]. Accordingly, we suspected that the role of RILs in irradiated microglia might resemble the regulation process in macrophages. Therefore, the classical inflammatory pathways, NF- $\mathrm{kB}$ and MAPK, were evaluated [26, 27]. We found that activation of NF-KB, JNK, or p38 pathways regulated the expression of selected RILs, which in turn influenced inflammation by regulating the phosphorylation levels of critical signaling proteins.

The DDR is the second important molecular feature of radiation-induced senescent microglia. Our results showed that the severity of the DDR was affected by radiation energy. The amount of energy that an ionizing particle transfers to the material traversed per unit distance is defined as the LET. High LET tends to mediate direct radiation interaction damage, while low LET mainly mediates indirect oxidative damage via water radiolysis [28, 46]. Unrepaired or inaccurately repaired DNA damage can induce cells to activate the death mechanism or acquire other states, such as senescence, which lead to permanent functional changes [47]. $\gamma \mathrm{H} 2 \mathrm{AX}$ is the most sensitive marker to examine the DDR [48]. Distinct expression profiles of RILs were associated with different levels of $\gamma \mathrm{H} 2 \mathrm{~A}$.X upregulation induced using different IR energies. In addition to key protein-coding genes, a subset of non-coding RNAs is also required for the cellular DDR. To coordinate the non-coding RNA-mediated DDR, DNA damage can regulate the expression levels of non-coding RNAs. In turn, lncRNAs participate in regulating certain steps of the DDR process, including recognition of DNA damage, signal relays, and the initiation of the repair [49]. Similarly, our result showed that the silencing of ENSMUST00000130679 resulted in decreased $\gamma \mathrm{H} 2 \mathrm{~A} . \mathrm{X}$ levels. This suggested that ENSMUST00000130679 participates in the DDR process. These findings will contribute to a better understanding of the reciprocal relationship between the radiation-induced DDR and RILs.

We detected the metabolism-related genes aggregation using RNA-seq and the accumulation of LDs in the early stage of microglia irradiation. A recent study confirmed that the accumulation of LDs in microglia is a marker of a dysfunctional state of the aging brain [30]. Furthermore, elevated ROS levels, followed by mitochondrial dysfunction, cause lipid peroxidation, which triggers the accumulation of LDs in glia. Reducing LD accumulation 
and lipid peroxidation in glia significantly delayed the onset of neurodegeneration [50]. Interestingly, we found that the accumulation of LDs decreased when lipogenesis was blocked in irradiated microglia. This suggested that enhancing lipogenesis, rather than inhibiting lipolysis, is the main cause of LD accumulation in irradiated microglia. Therefore, we further evaluated the roles of RILs in lipogenesis of irradiated microglia. The upregulation of several RILs after IR was significantly attenuated when lipogenesis was blocked, indicating that certain RILs are involved in lipogenesis. Given that most IncRNAs have varying degrees of overlap with nearby protein-coding genes, compared with the lncRNA itself, the transcriptional activity within the locus containing the IncRNA is more likely to be the source of functional regulation [31]. Therefore, it is reasonable to hypothesize that RILs regulate lipogenesis via nearby genes. Using GO analysis, we showed that 79 out of 226 metabolism-related genes were involved in lipid metabolism. Surprisingly, over half of them were related to lipogenesis. Although we could not conclude directly which lipogenesis-related genes are affected by the RILs, we could speculate that the RILs regulate LD accumulation at least partly via their effects on nearby lipogenesis-related genes.

As the first-line defense against injury, intact microglia undergo "beneficial activation" in the context of brain injury, in which they migrate to the injury, phagocytose the debris, secrete various cytokines and chemoattractants, and eliminate inflammation, eventually resulting in tissue recovery. However, once the activation passes the threshold of beneficial function and becomes deleterious, microglia swiftly acquire a neurotoxic phenotype, causing impairment to phagocytosis and synaptic plasticity, the generation of ROS, and inflammatory disorder, which together contribute to neuronal damage or death [6]. To test the impact of irradiated-induced senescent microglia on neurons, we built a co-culture system of BV2 and HT22 cells. The two selected RILs had a significant impact on the apoptosis ratio of HT22 cells, which suggested that RILs might be novel prognostic biomarkers and potential targets for protection against encephalopathy.

\section{Conclusions}

In summary, our study provides new insights into the pathophysiology of irradiated microglia. Inflammation, the DDR, and metabolic changes are the three principal molecular features of senescence in irradiated microglia, and distinct RILs are closely associated with these molecular features. Increased understanding of the functions of RILs will reveal their potential therapeutic value in RIBI.

\section{Supplementary Information}

The online version contains supplementary material available at https://doi. org/10.1186/s12974-020-02001-1.

Additional file 1: Supplementary Table 1. qRT-PCR primers used.

\section{Abbreviations}

RIBI: Radiation-induced brain injury; IncRNAs: Long noncoding RNAs; DDR: DNA damage and response; RILs: Radiation-induced differentially expressed IncRNAs; IR: lonizing radiation; QoL: Quality of life;

DAMPs: Damage-associated molecular patterns; MMP: Matrix metalloproteases; LDs: Lipid droplets; FC: Fold-change; OA: Oleic acid; D-

PBS: Dulbecco's phosphate-buffered saline; LPS: Lipopolysaccharide; ISH: In situ hybridization; SSC: Saline sodium citrate; SA- $\beta$-Gal: Senescence-associated $\beta$-galactosidase; Iba-1: Ionized calcium-binding adapter molecule 1; Linac: Linear accelerator; Dpi: Days post-irradiation; RNA-seq: RNAsequencing; FDR: False discovery rate; GO: Gene ontology; KEGG: Kyoto Encyclopedia of Genes and Genomes; CDKi: Cyclin-dependent kinase inhibitor; Jnk: C-jun N-terminal kinase; Erk: Extracellular signal-regulated kinase; IL-1 $\beta$ : Interleukin-1 $\beta$; TNFa: Tumor necrosis factor $a$; LET: Linear energy transfer; SREBP: Sterol regulatory element-binding protein; ROS: Reactive oxygen species; ELISA: Enzyme-linked immunosorbent assay; qRTPCR: Quantitative real-time reverse transcription-polymerase chain reaction

\section{Acknowledgements}

Not applicable.

\section{Authors' contributions}

YWY, ZXM, and AMJ designed and supervised this research. HFJ and ABR performed the cell culture experiments. XAA and ABR performed the isolation of primary microglia and senescence assays. QXZ performed the quality control for the IR treatment. ZH, BYW, JLZ, and XYC performed the qRT-PCR, western blotting, and ELISA experiments. YHT and LR performed the immunofluorescence and in situ hybridization experiments. NYZ, MZL, and RHZ performed the data analysis. AAX and RL were major contributors to writing the manuscript. All authors read and approved the final manuscript.

\section{Funding}

This study was funded by the National Natural Science Foundation of China (Grant numbers: 81773354, 81872195, and 81803170), the Project of Educational Commission of Guangdong Province of China (Grant number: 2017KTSCX152). The funding provided the resources for the study design, data collection, data analysis, and manuscript polishing. The funding bodies had no role in the design of the study; the collection, analysis, and interpretation of the data; or in writing the manuscript.

Availability of data and materials

All data in this manuscript are available on reasonable request.

Ethics approval and consent to participate

All animal experiments were approved by the Institutional Animal Care and Use Committee at Guangzhou Medical University.

Consent for publication

Not applicable.

Competing interests

The authors declare that they have no competing interests.

\section{Author details}

${ }^{1}$ Department of Radiation Oncology, Affiliated Cancer Hospital \& Institute of Guangzhou Medical University, No 78, Hengzhigang Road, Yuexiu District, Guangzhou 510095, Guangdong, People's Republic of China. ${ }^{2}$ Department of Radiation Oncology, Sun Yat-Sen University Cancer Center, Guangzhou, People's Republic of China. ${ }^{3}$ Department of Pharmacology, Emory University School of Medicine, Atlanta, GA, USA. 
Received: 20 January 2020 Accepted: 16 October 2020 Published online: 28 October 2020

\section{References}

1. Greene-Schloesser D, Moore E, Robbins ME. Molecular pathways: radiationinduced cognitive impairment. Clin Cancer Res. 2013;19:2294-300.

2. Bond SM, Dietrich MS, Gilbert J, Ely EW, Jackson JC, Murphy BA. Neurocognitive function in patients with head and neck cancer undergoing primary or adjuvant chemoradiation treatment. Support Care Cancer. 2016;24:4433-42.

3. Genc M, Genc E, Genc BO, Kiresi DA. Significant response of radiation induced CNS toxicity to high dose steroid administration. Br J Radiol. 2006; 79:e196-9.

4. Rapp SR, Case LD, Peiffer A, Naughton MM, Chan MD, Stieber W, et al. Donepezil for Irradiated Brain Tumor Survivors: A Phase III Randomized Placebo-Controlled Clinical Trial. J Clin Oncol. 2015;33:1653-9.

5. Brown PD, Pugh S, Laack NN, Wefel JS, Khuntia D, Meyers C, et al. Memantine for the prevention of cognitive dysfunction in patients receiving whole-brain radiotherapy: a randomized, double-blind, placebo-controlled trial. Neuro Oncol. 2013;15:1429-37.

6. Block ML, Zecca L, Hong JS. Microglia-mediated neurotoxicity: uncovering the molecular mechanisms. Nat Rev Neurosci. 2007:8:57-69.

7. Xu P, Xu Y, Hu B, Wang J, Pan R, Murugan M, et al. Extracellular ATP enhances radiation-induced brain injury through microglial activation and paracrine signaling via P2X7 receptor. Brain Behav Immun. 2015;50:87-100.

8. Garaschuk O, Semchyshyn HM, Lushchak VI. Healthy brain aging: interplay between reactive species, inflammation and energy supply. Ageing Res Rev. 2018;43:26-45

9. Baker DJ, Petersen RC. Cellular senescence in brain aging and neurodegenerative diseases: evidence and perspectives. J Clin Invest. 2018; 128:1208-16.

10. Flanary B. The role of microglial cellular senescence in the aging and Alzheimer diseased brain. Rejuvenation Res. 2005;8:82-5.

11. Childs BG, Durik M, Baker DJ, van Deursen JM. Cellular senescence in aging and age-related disease: from mechanisms to therapy. Nature Medicine. 2015;21:1424-35.

12. Le O, Palacio L, Bernier G, Batinic-Haberle I, Hickson G, Beausejour C. INK4a/ ARF Expression Impairs Neurogenesis in the Brain of Irradiated Mice. Stem Cell Reports. 2018;10:1721-33.

13. Schneider L, Pellegatta S, Favaro R, Pisati F, Roncaglia P, Testa G, et al. DNA damage in mammalian neural stem cells leads to astrocytic differentiation mediated by BMP2 signaling through JAK-STAT. Stem Cell Reports. 2013;1:123-38.

14. Turnquist C, Beck JA, Horikawa I, Obiorah IE, Von Muhlinen N, Vojtesek B, et al. Radiation-induced astrocyte senescence is rescued by Delta133p53. Neuro Oncol. 2019;21:474-85.

15. McRobb LS, McKay MJ, Gamble JR, Grace M, Moutrie V, Santos ED, et al. lonizing radiation reduces ADAM10 expression in brain microvascular endothelial cells undergoing stress-induced senescence. Aging (Albany NY). 2017:9:1248-68

16. Li MD, Burns TC, Kumar S, Morgan AA, Sloan SA, Palmer TD. Aging-like changes in the transcriptome of irradiated microglia. Glia. 2015;63:754-67.

17. Goodrich JA, Kugel JF. Non-coding-RNA regulators of RNA polymerase II transcription. Nat Rev Mol Cell Biol. 2006;7:612-6.

18. Abdelmohsen K, Panda A, Kang MJ, Xu J, Selimyan R, Yoon JH, et al. Senescence-associated IncRNAs: senescence-associated long noncoding RNAs. Aging Cell. 2013;12:890-900.

19. Hofmann P, Sommer J, Theodorou K, Kirchhof L, Fischer A, Li Y, et al. Long non-coding RNA H19 regulates endothelial cell aging via inhibition of STAT3 signalling. Cardiovasc Res. 2019;115:230-42.

20. Li Z, Chao TC, Chang KY, Lin N, Patil VS, Shimizu C, et al. The long noncoding RNA THRIL regulates TNFalpha expression through its interaction with hnRNPL. Proc Natl Acad Sci USA. 2014;111:1002-7.

21. Nojima T, Tellier M, Foxwell J, Ribeiro de Almeida C, Tan-Wong SM, Dhir S, et al. Deregulated expression of mammalian IncRNA through loss of SPT6 induces R-Loop formation, replication stress, and cellular senescence. Mol Cell. 2018;72:970-84 e977.

22. Sun LY, Li XJ, Sun YM, Huang W, Fang K. Han Cet al. LncRNA ANRIL regulates $A M L$ development through modulating the glucose metabolism pathway of AdipoR1/AMPK/SIRT1. Mol Cancer. 2018;17:127.

23. Childs BG, Gluscevic M, Baker DJ, Laberge RM, Marquess D, Dananberg J, et al. Senescent cells: an emerging target for diseases of ageing. Nat Rev Drug Discov. 2017;16:718-35.
24. Young AR, Narita M. SASP reflects senescence. EMBO Rep. 2009;10:228-30

25. Balentova S, Adamkov M. Molecular, cellular and functional effects of radiation-induced brain injury: a review. Int J Mol Sci. 2015;16:27796-815.

26. Xue J, Dong JH, Huang GD, Qu XF, Wu G, Dong XR. NF-kappaB signaling modulates radiation induced microglial activation. Oncol Rep. 2014;31:2555-60.

27. Deng Z, Sui G, Rosa PM, Zhao W. Radiation-induced c-Jun activation depends on MEK1-ERK1/2 signaling pathway in microglial cells. PLoS One. 2012:7:e36739.

28. Azzam El, Jay-Gerin JP, Pain D. Ionizing radiation-induced metabolic oxidative stress and prolonged cell injury. Cancer Lett. 2012;327:48-60.

29. $\mathrm{Hu} X, X u B, G e W$. The role of lipid bodies in the microglial aging process and related diseases. Neurochem Res. 2017;42:3140-8.

30. Marschallinger J, Iram T, Zardeneta M, Lee SE, Lehallier B, Haney MS, et al. Lipid-droplet-accumulating microglia represent a dysfunctional and proinflammatory state in the aging brain. Nat Neurosci. 2020;23:194-208.

31. Kopp F, Mendell JT. Functional classification and experimental dissection of long noncoding RNAs. Cell. 2018;172:393-407.

32. Baker DJ, Childs BG, Durik M, Wijers ME, Sieben CJ, Zhong J, et al. Naturally occurring p16(Ink4a)-positive cells shorten healthy lifespan. Nature. 2016:530:184-9.

33. Schneider L, Fumagalli M, d'Adda di Fagagna F. Terminally differentiated astrocytes lack DNA damage response signaling and are radioresistant but retain DNA repair proficiency. Cell Death Differ. 2012:19:582-91.

34. Jacobs JJ, de Lange T. Significant role for p16INK4a in p53-independent telomere-directed senescence. Curr Biol. 2004;14:2302-8.

35. Mangold CA, Wronowski B, Du M, Masser DR, Hadad N, Bixler GV, et al. Sexually divergent induction of microglial-associated neuroinflammation with hippocampal aging. J Neuroinflammation. 2017;14:141-60.

36. Krukowski K, Grue K, Frias ES, Pietrykowski J, Jones T, Nelson G, et al. Female mice are protected from space radiation-induced maladaptive responses. Brain Behav Immun. 2018;74:106-20.

37. Hinkle JJ, Olschowka JA, Love TM, Williams JP, O'Banion MK. Cranial irradiation mediated spine loss is sex-specific and complement receptor-3 dependent in male mice. Sci Rep. 2019;9:18899.

38. Greene-Schloesser D, Robbins ME. Radiation-induced cognitive impairment—from bench to bedside. Neuro Oncol. 2012;14(Suppl 4):iv37-44.

39. Rodier F, Munoz DP, Teachenor R, Chu V, Le O, Bhaumik D, et al. DNA-SCARS distinct nuclear structures that sustain damage-induced senescence growth arrest and inflammatory cytokine secretion. J Cell Sci. 2011;124:68-81.

40. Ding F, Yao J, Rettberg JR, Chen S, Brinton RD. Early decline in glucose transport and metabolism precedes shift to ketogenic system in female aging and Alzheimer's mouse brain: implication for bioenergetic intervention. PLoS One. 2013:8:e79977.

41. Kour S, Rath PC. Long noncoding RNAs in aging and age-related diseases Ageing Res Rev. 2016;26:1-21.

42. Ng SY, Lin L, Soh BS, Stanton LW. Long noncoding RNAs in development and disease of the central nervous system. Trends Genet. 2013;29:461-8.

43. Krawczyk M. Emerson BM. p50-associated COX-2 extragenic RNA (PACER) activates COX-2 gene expression by occluding repressive NF-kappaB complexes. Elife. 2014;3:e01776.

44. Du M, Yuan L, Tan X, Huang D, Wang X, Zheng Z, et al. The LPS-inducible IncRNA Mirt2 is a negative regulator of inflammation. Nat Commun. 2017;8:2049.

45. Atianand MK, Hu W, Satpathy AT, Shen Y, Ricci EP, Alvarez-Dominguez JR, et al. A long noncoding RNA lincRNA-EPS acts as a transcriptional brake to restrain inflammation. Cell. 2016;165:1672-85.

46. Jezkova L, Zadneprianetc M, Kulikova E, Smirnova E, Bulanova T, Depes D, et al. Particles with similar LET values generate DNA breaks of different complexity and reparability: a high-resolution microscopy analysis of gamma H2AX/53BP1 foci. Nanoscale. 2018;10:1162-79.

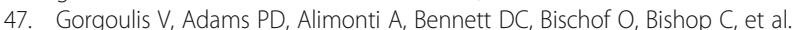
Cellular senescence: defining a path forward. Cell. 2019;179:813-27.

48. Sharma A, Singh K, Almasan A. Histone H2AX phosphorylation: a marker for DNA damage. Methods Mol Biol. 2012;920:613-26.

49. Zhang C, Peng G. Non-coding RNAs: an emerging player in DNA damage response. Mutat Res Rev Mutat Res. 2015;763:202-11.

50. Liu L, Zhang K, Sandoval H, Yamamoto S, Jaiswal M, et al. Glial lipid droplets and ROS induced by mitochondrial defects promote neurodegeneration. Cell. 2015;160:177-90.

\section{Publisher's Note}

Springer Nature remains neutral with regard to jurisdictional claims in published maps and institutional affiliations. 